\title{
Rheological and Thermal Properties of Poly(vinyl alcohol)-Ethylene Glycol-Water Gels
}

\author{
Katsuyoshi Nishinari ${ }^{\dagger}$ and Mineo WATASE* \\ National Food Research Institute, Tsukuba, Ibaraki 305, Japan \\ * Faculty of Liberal Arts, Shizuoka University, Ohya, Shizuoka 422, Japan
}

(Received July 29, 1992)

\begin{abstract}
Effects of ethylene glycol (EG) on rheological and thermal properties of poly(vinyl alcohol) (PVA) gels were studied by longitudinal vibration measurements and differential scanning calorimetry. Elastic modulus of PVA gels as a function of EG content showed a maximum around $0.35 \mathrm{~mol}$ fraction ( $\mathrm{mf}$ ) EG, while the melting temperature and the heat absorbed on forming one mol of junction zones increased monotonously with increasing EG content. It was suggested that junction zones of PVA gels become more heat resistant by the addition of EG, however, the number of junction zones becomes maximum around $0.35 \mathrm{mf}$ EG. The results were compared with those for PVA-dimethyl sulfoxide-water gels reported previously.

KEY WORDS Poly(vinyl alcohol) / Gel / Differential Scanning Calorimetry / Rheology / Ethylene Glycol /
\end{abstract}

It is well-known that poly(vinyl alcohol) (PVA) aqueous solution does not form a strong gel, and its rheological properties change remarkably with time lapse. ${ }^{1}$ Repeating cycles of freeze-thaw ${ }^{2-5}$ and mixing with dimethyl sulfoxide (DMSO) ${ }^{6,7}$ make a strong gel of PVA. Ethylene glycol (EG) changes the structure of water, and also interacts with hydroxyl groups in gel forming polymer such as agarose. Rheological and thermal properties of agarose gels were found to be influenced strongly by the addition of EG. ${ }^{8,9}$

Recently, a zipper model approach was proposed to understand the relationship between the structure of junction zones and the heat capacity of thermo-reversible gels. ${ }^{10}$ According to the zipper model approach, the heat capacity $C$ of a gel consisting of $\mathscr{N}$ zippers is written as

$$
\frac{C}{k}=\mathscr{N}\left(\log \frac{G}{x}\right)^{2}\left[\frac{2 x}{(1-x)^{2}}\right.
$$

$$
\left.+\frac{N(N+1) x^{N}\left[-x^{N+1}+(N+1) x-N\right]}{\left[1-(N+1) x^{N}+N x^{N+1}\right]^{2}}\right] .
$$

where $N$ is the number of parallel links in a single zipper, $\varepsilon$ is the energy required to open a link, $G$ is the rotational freedom of a link, $x=G \exp (-\varepsilon / k T)$, and $T$ is the absolute temperature. In a differential scanning calorimetry (DSC) measurement, the heat flow difference $\mathrm{d} q / \mathrm{d} t$ between sample and reference is recorded as a function of temperature. Here, $q$ and $t$ represent the heat and time respectively. Since $\mathrm{d} q=C \mathrm{~d} T$, or $\mathrm{d} q / \mathrm{d} t=C \mathrm{~d} T / \mathrm{d} t$, the number $\mathcal{N}$ represents the number of zippers per total mass of the gel sample. Since eq 1 is based on equilibrium statistical thermodynamics, it is necessary to choose the scanning rate $\mathrm{d} T / \mathrm{d} t$ as slow as possible.

${ }^{\dagger}$ Present address: Faculty of Science of Living, Osaka City University, Sumiyoshi, Osaka 558, Japan. 


\section{EXPERIMENTAL}

\section{DSC Measurements}

DSC measurements were done with a Seiko DSC 10 calorimeter. Approximately $45 \mathrm{mg}$ of the sample was sealed into the DSC pan hermetically, and the reference pan was filled with exactly the same amount
$( \pm 0.1 \mathrm{mg}$ ) of solvent. The temperature was raised from $5^{\circ} \mathrm{C}$ to $140^{\circ} \mathrm{C}$ at $2^{\circ} \mathrm{C} \mathrm{min}^{-1}$, and lowered at the same rate. To examine the state of water in gels, the temperature was lowered by liquid nitrogen to $-120^{\circ} \mathrm{C}$, and kept for $10 \mathrm{~min}$, and then raised at $2^{\circ} \mathrm{Cmin}^{-1}$.
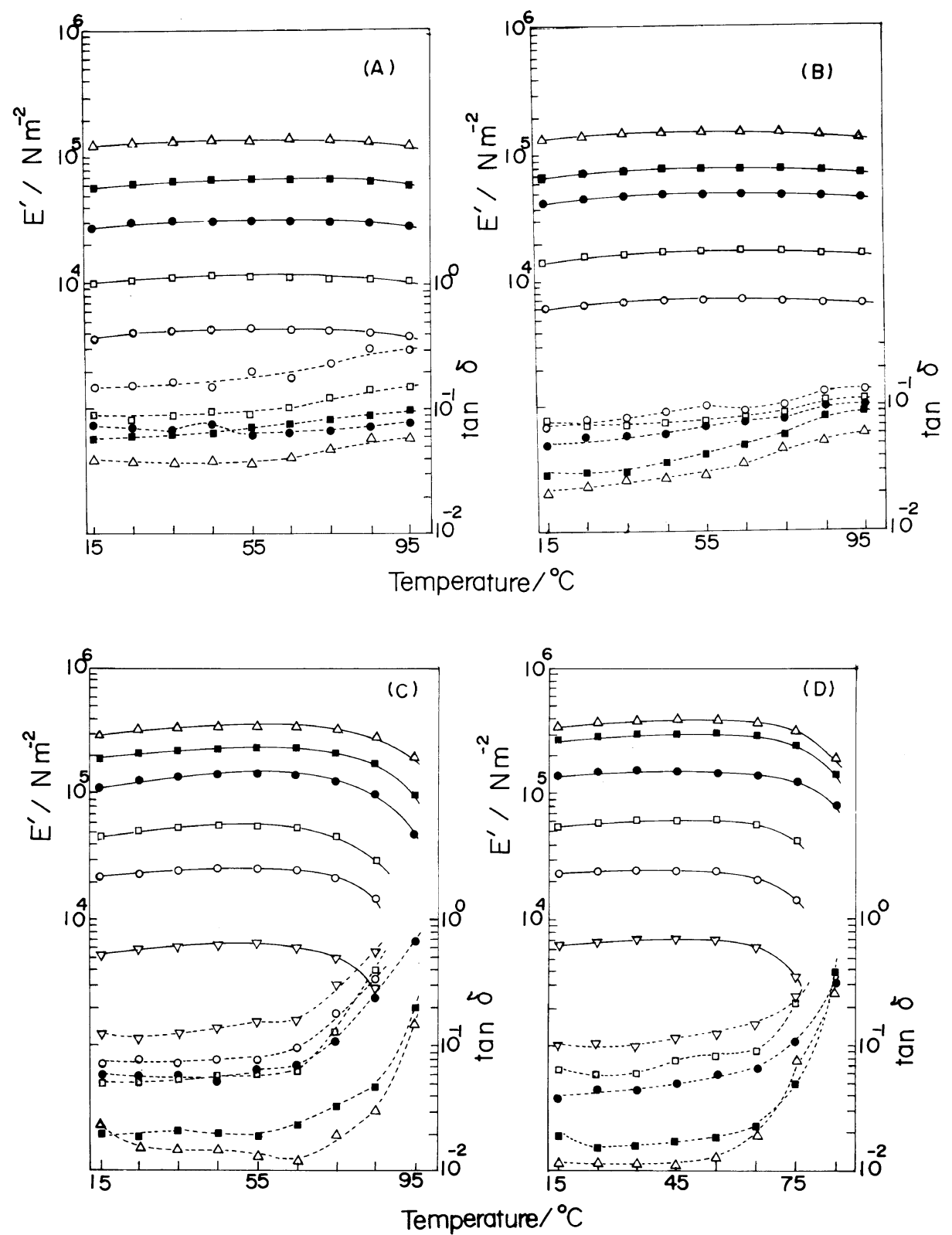


\section{Rheological Measurements}

Preparation of cylindrically molded gels was described previously. ${ }^{2,3,6}$ The gels were kept at $4{ }^{\circ} \mathrm{C}$ for three days and then at the measurement temperature for $30 \mathrm{~min}$. The cylindrical gels were subjected to longitudinal vibrations of $2.5 \mathrm{~Hz}$. Young's storage and loss moduli were determined at once by a phase sensitive circuit.

\section{PVA Samples}

The degree of polymerization $(D P)$ and the degree of saponification $(D S)$ for PVA used in this study were 1700 and $99.9 \mathrm{~mol} \%$ respectively. The PVA samples were given by Kuraray Co. Ethylene glycol of a reagent

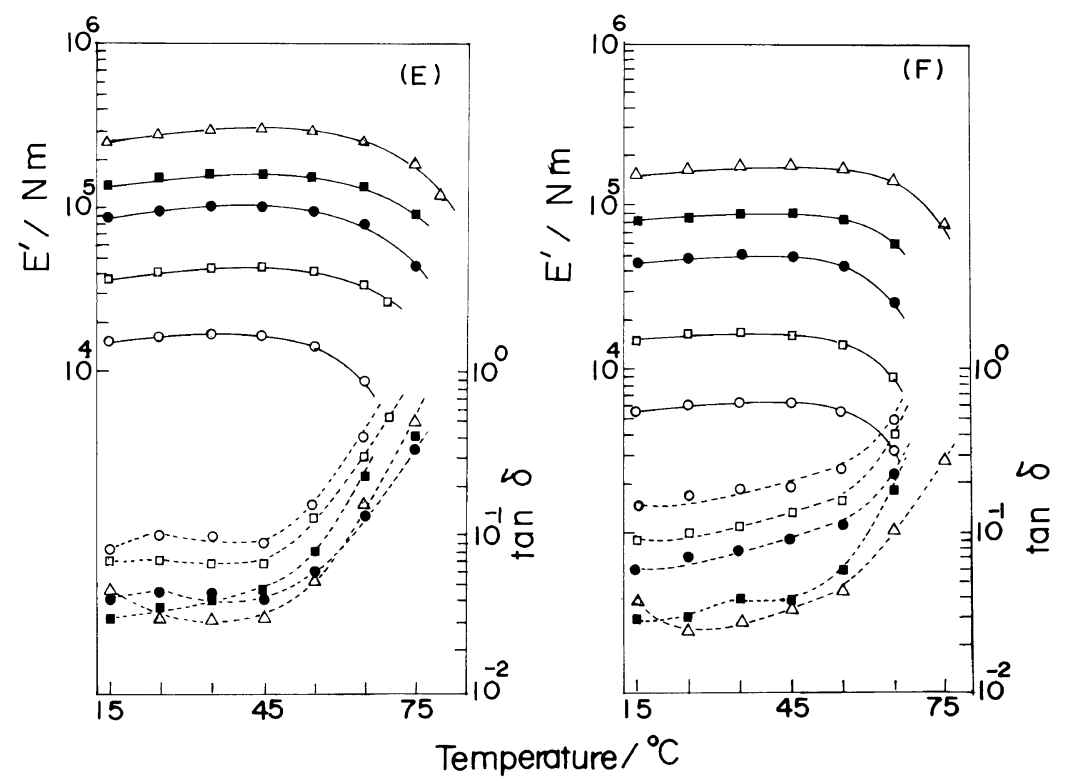

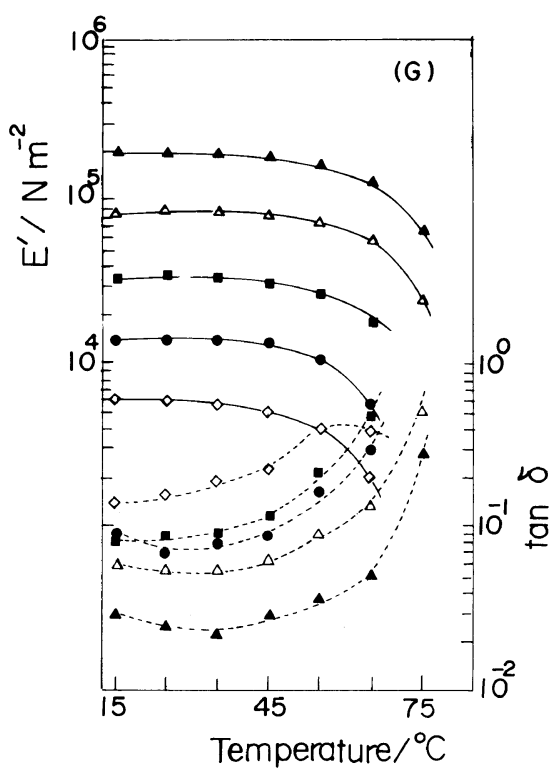

Figure 1. Temperature dependence of the storage Young's modulus $E^{\prime}$ (solid curve) and mechanical loss tangent $\tan \delta$ (broken curve) for PVA-EG-water gels, PVA concentrations: $\Delta, 20 \mathrm{wt} \% ; \triangle, 15 \mathrm{wt} \% ; \boldsymbol{\square}, 12 \mathrm{wt} \% ; \boldsymbol{O}, 10 \mathrm{wt} \%$; $\diamond, 8 \mathrm{wt} \% ; \square, 7 \mathrm{wt} \% ; \bigcirc, 5 \mathrm{wt} \% \nabla, 3 \mathrm{wt} \%$. EG contents: (A) $1 \mathrm{mf}$; (B) $0.794 \mathrm{mf}$; (C) $0.458 \mathrm{mf}$; (D) $0.321 \mathrm{mf}$; (E) $0.222 \mathrm{mf}$; (F) $0.147 \mathrm{mf}$; (G) $0.087 \mathrm{mf}$. 
grade (Wako Junyaku Kogyo Co.) was used without further purification.

\section{RESULTS AND DISCUSSION}

Figures 1(A)-(G) show the temperature dependence of storage Young's modulus $E^{\prime}$ and mechanical loss tangent $\tan \delta$ for PVAEG-water gels with various PVA concentrations, and various EG contents. $E^{\prime}$ as a function of temperature was almost independent of temperature for PVA gels with $1 \mathrm{mf}$ EG (Figure 1(A)), $0.794 \mathrm{mf}$ EG (Figure 1(B)) up to $85^{\circ} \mathrm{C}$, and $\tan \delta$ slightly increased. Values of $\tan \delta$ were smaller for concentrated gels than for dilute gels. $E^{\prime}$ for PVA gels with $0.458 \mathrm{mf}$ EG (Figure 1(C)), $0.321 \mathrm{mf}$ EG (Figure 1(D)), $0.222 \mathrm{mf}$ EG (Figure 1(E)), $0.147 \mathrm{mf}$ EG (Figure 1(F)) increased gradually up to $55^{\circ} \mathrm{C}$ and then decreased with increasing temperature. At the temperature range where $E^{\prime}$ decreased rapidly around $65^{\circ} \mathrm{C}, \tan \delta$ increased steeply indicating the commencement of internal melting of PVA gels; this is caused by the release of PVA molecular chains from the junction zones in gels consisting of the aggregation of extended PVA molecules. ${ }^{11,12}$ The schematic representation of PVA gel structure in water and DMSO/water consisting of junction zones and flexible chains has been proposed again recently. ${ }^{13} E^{\prime}$ for PVA gels with a very low EG content $(0.087 \mathrm{mf}$, Figure $1(\mathrm{G}))$ decreased gradually up to $55^{\circ} \mathrm{C}$ and then decreased rapidly beyond this temperature with increasing temperature.

The fact that $E^{\prime}$ of PVA gels containing EG showed a maximum at the EG content between $0.321 \mathrm{mf}$ and $0.458 \mathrm{mf}$ is understood as follows. In the presence of higher EG content than $0.458 \mathrm{mf}$, the gelation was inhibited by free EG, and in the lower EG content, the gelation was inhibited by free water. As will be discussed later, gels consist of the balance between the solubility and the crystallinity. In the presence of excessive water, free water molecules solubilize PVA and shift this balance to the solubility side. The elastic modulus of thermo-reversible gels depends on the number and the structure of cross-linking junction zones, and on the conformation of flexible chains connecting these junction zones. A simple model, called as "reel-chain model" consisting of Langevin chains whose both ends are loosely fixed in crystalline junction regions in such a way that the segments near each end are bound densely in one of the junction region, has been proposed in order to explain the temperature dependence of elasticity of thermoreversible gels. ${ }^{14}$ According to this reelchain model, the segments are assumed to be released from the junction zone with increasing temperature just as thread is released from a reel. When all the segments in one of both ends are liberated from the junction zone, the chain is called a free chain in the theory of rubber elasticity, and ceases to contribute to the elasticity. ${ }^{15}$ Using this model, the elastic modulus of thermoreversible gels can be calculated numerically by assuming the number $\mathscr{N}$ of molecular chains in a unit volume, the values of bonding energy $\varepsilon$, average end-to-end distance of Langevin chains $r_{\mathrm{m}}$, an upper limit $v$ of the number of segments released from the junction zones before the chain ceases to contribute to the elasticity. It is, however, not possible to determine these parameters uniquely only from viscoelastic measurements. Therefore, it is desirable to combine the rheological study with other methods such as neutron or X-ray small angle scattering. It is still possible to estimate qualitatively whether these parameters increase or decrease by the change in environmental conditions. The number of cross-linking junction zones will decrease with increasing water content. The elastic modulus of thermo-reversible gels is proportional to the number of cross-linking junction zones if the conformation of flexible chains remains the 


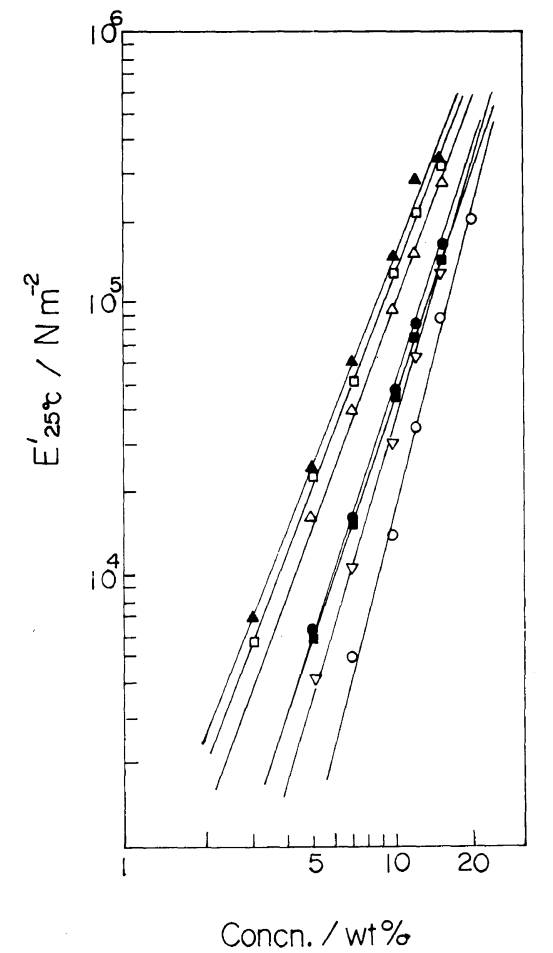

Figure 2. Double logarithmic plot of $E^{\prime}$ for PVA gels with various $\mathrm{EG}$ contents at $25^{\circ} \mathrm{C} v s$. the concentration of PVA. EG contents: $\bigcirc, 0.087 \mathrm{mf} ; 0.147 \mathrm{mf} ; \triangle$, $0.222 \mathrm{mf} ; \Delta, 0.321 \mathrm{mf} ; \square, 0.458 \mathrm{mf} ; \square, 0.794 \mathrm{mf} ; \nabla$, $1 \mathrm{mf}$.

same. The presence of EG changes the ratio of entropic and energetic contributions to the elastic modulus. The entropic term increases with increasing length of flexible chains as was found in agarose gels. ${ }^{16} \mathrm{At}$ a certain EG content between 0.321 and $0.458 \mathrm{mf}$, three main factors, the number and structure of cross-linking junction zones, the conformation of flexible chains connecting junction zones, or $\varepsilon, r_{\mathrm{m}}, v$, and $\mathcal{N}$ in the above-mentioned model which determine the elastic modulus become optimum to realize the maximum of elastic modulus.

Figure 2 shows the double logarithmic plot of $E^{\prime}$ for PVA gels with various EG contents at $25^{\circ} \mathrm{C} v s$. the PVA concentration. Straight lines were obtained from the least squares method. The exponent $n$ in the

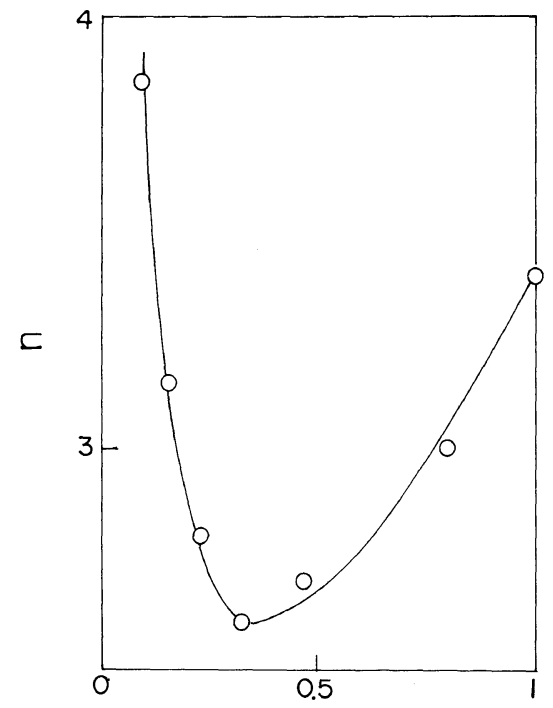

Ethylene glycol concn./mf

Figure 3. The relation between the exponent $n$ in the equation $E^{\prime} \sim(\text { concn })^{n}$ for PVA gels and EG content.

equation $E^{\prime} \sim(\text { concn })^{n}$ was determined from the slope of these straight lines, and is shown as a function of EG content in Figure 3. The value of $n$ becomes minimum at around $0.32 \mathrm{mf}$ EG. Concentration dependence of elastic modulus of the gels has been discussed by a cascade treatment of Clark and Ross-Murphy ${ }^{17}$ and by a modified rubber elasticity theory of Oakenfull. ${ }^{18}$ Both theories predict that the slope in the double logarithmic plot of the elastic modulus against the reduced polymer concentration $C / C_{0}$, where $C_{0}$ is the critical concentration below which no gelation occurs, is steeper in dilute region than in concentrated region. ${ }^{19}$ The fact that the exponent $n$ becomes minimum around $0.32 \mathrm{mf}$ EG suggests that the critical concentration $C_{0}$ becomes minimum at around $0.32 \mathrm{mf}$ EG. The effects of EG on the conformation of PVA molecules in water, and on the structure of junction zones should be determined so that we may be able to understand further details in the relation between structure and gelling prop- 
erties.

Upper curves in Figure 4(A) show heating DSC curves for PVA gels with various PVA concentrations and various EG contents. An endothermic peak accompanying gel-to-sol transition appeared around $120^{\circ} \mathrm{C}$, and the peak temperature shifted to higher temperatures with increasing concentration of PVA. This endothermic peak temperature will be denoted as $T_{\mathrm{m}}$ (melting temperature) here- after. In addition to this main endothermic peak around $120^{\circ} \mathrm{C}$, an endothermic shoulder was observed around $100^{\circ} \mathrm{C}$, and it became more conspicuous with increasing concentration of PVA. Lower curves in Figure 4(A) are cooling DSC curves for PVA-EG-water systems with various PVA concentrations and various EG contents. A sharp exothermic peak accompanying sol-to-gel transition appeared around $70^{\circ} \mathrm{C}$ which is far lower
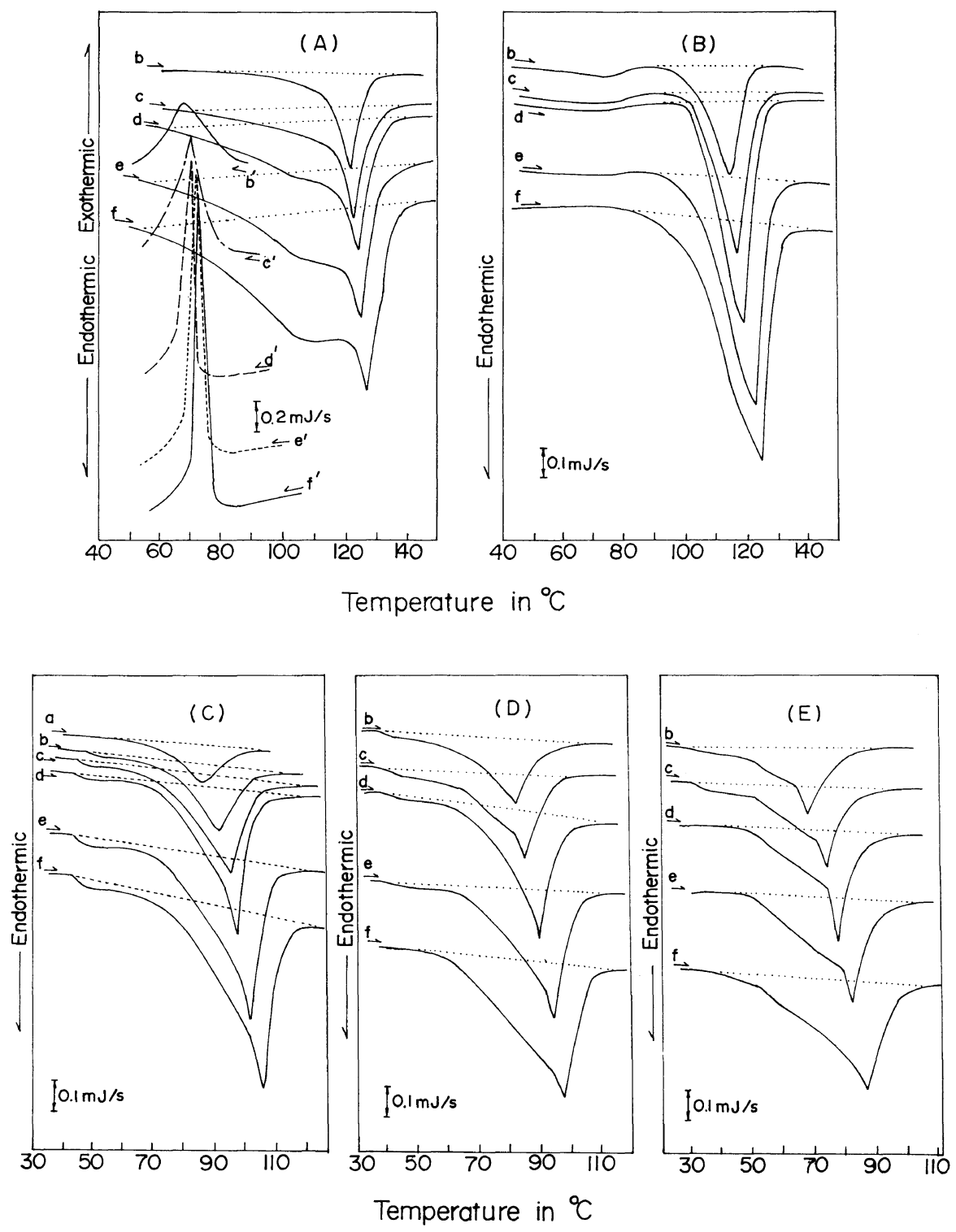
than $T_{\mathrm{m}}$. The exothermic peak temperature will be called $T_{\mathrm{s}}$ (setting temperature) hereafter.

The reason why $T_{\mathrm{m}}$ in a heating DSC curve appears at a higher temperature than $T_{\mathrm{s}}$ in its cooling DSC curve is explained by the zipper model approach as follows: when the temperature is raised, the rotational freedom $G$ of a parallel link which forms a zipper would start from the lower value $G_{\mathrm{g}}$ corresponding to the gel state. Then, the gel will expand, which will give rise to an increase in the rotational freedom. On the contrary, when the temperature is lowered from higher temperatures than the melting point, $G$ will start from the higher value $G_{\text {s }}$ corresponding to the sol state. Therefore, the opening of molecular zippers begins to occur at small $G$ values in heating process, while gelation by cooling will take place with decreasing $G$ which starts from large $G$ values at higher temperatures. Then, the average effective value of $G$ is small in heating and is large in cooling. As a first approximation, therefore, we can say that the melting peak temperature $T_{\mathrm{m}}$ is deter- mined by a certain average $\bar{G}_{\mathrm{g}}$ of $G$ for gel state and the setting temperature $T_{\mathrm{s}}$ is determined by an average $\bar{G}_{\mathrm{s}}$ of $G$ for sol state. Apparently, $\bar{G}_{\mathrm{g}}<\bar{G}_{\mathrm{s}}$, hence, $T_{\mathrm{m}}$ is expected to be higher than $T_{\mathrm{s}}$.

In the case of heating, the transition caused by the opening of the zippers will start as soon as the temperature arrives at the tail of the $\mathrm{C}-\mathrm{T}$ curve corresponding to $G=G_{\mathrm{g}}$. In cooling, on the contrary, the pair-wise coupling cannot start so easily because of the difficulty for a long molecule to find its partner in appropriate positions for the zipper construction. Hence a state like supercooling may take place in the course of cooling. It is, therefore, reasonable that the transition is sharper in cooling than in heating as has been observed for PVAEG-water gels (Figure 4(A)) and agarose and kappa-carrageenan gels. ${ }^{20}$

DSC heating curves of PVA gels with various concentrations and with various $E G$ contents are shown in Figures $4(\mathrm{~B})-(\mathrm{H})$. Generally, $T_{\mathrm{m}}$ shifted to higher temperatures with increasing concentration of PVA. $T_{m}$ of PVA gels of the same PVA concentra-
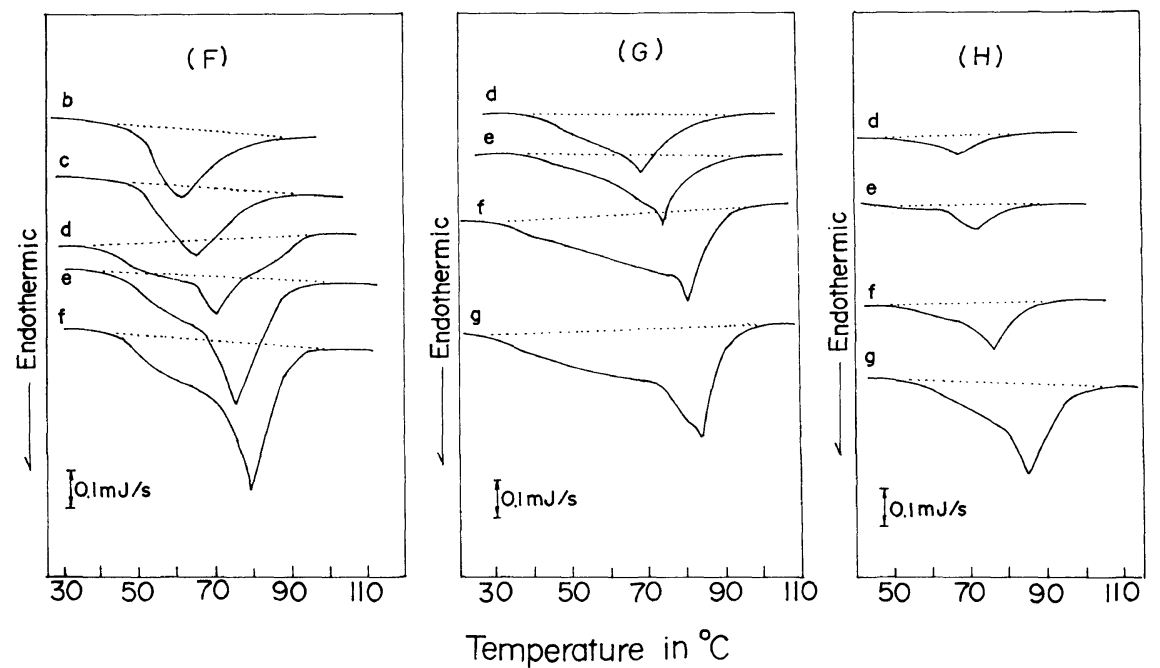

Figure 4. Heating and cooling DSC curves for PVA-EG-water systems. PVA concentrations: $a, a^{\prime}$, $3 \mathrm{wt} \%$; b, b', $5 \mathrm{wt} \%$; c,c', $7 \mathrm{wt} \%$; d, d' 9 wt \%; e, e', $12 \mathrm{wt} \% ; \mathrm{f}, \mathrm{f}^{\prime}, 15 \mathrm{wt} \% ; \mathrm{g}, \mathrm{g}^{\prime}, 20 \mathrm{wt} \%$. EG contents: (A) $1 \mathrm{mf}$; (B) $0.794 \mathrm{mf}$; (C) $0.458 \mathrm{mf}$; (D) $0.357 \mathrm{mf}$; (E) $0.222 \mathrm{mf}$; (F) $0.147 \mathrm{mf}$; (G) $0.087 \mathrm{mf}$; (H) $0.04 \mathrm{mf}$. 


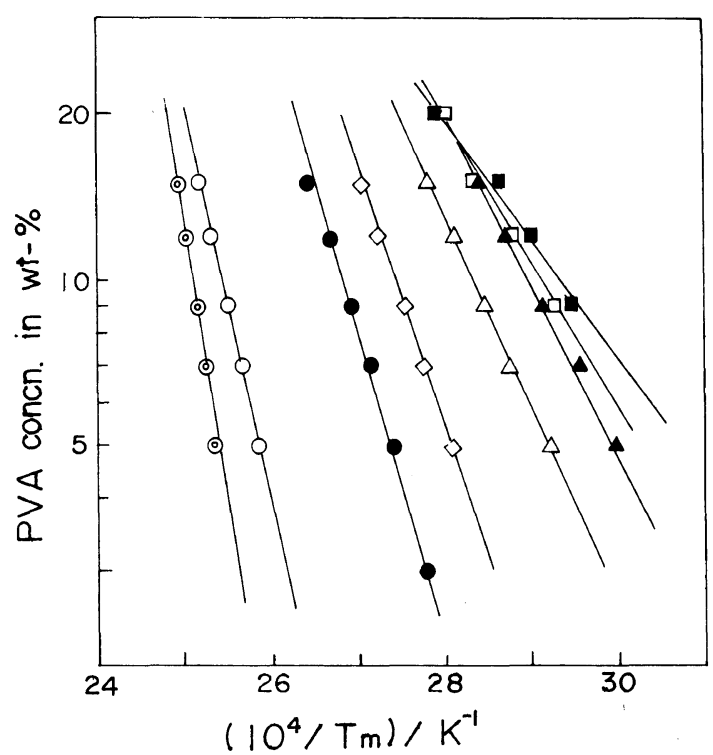

Figure 5. Eldridge-Ferry plot for PVA-EG-water gels. EG contents: $\odot, 1 \mathrm{mf} ; \bigcirc, 0.794 \mathrm{mf} ; \bigcirc, 0.458 \mathrm{mf}, \diamond$, $0.357 \mathrm{mf} ; \triangle, 0.222 \mathrm{mf} ; \mathbf{\Delta}, 0.147 \mathrm{mf} ; \square, 0.087 \mathrm{mf}, \square$, $0.04 \mathrm{mf}$.

tion shifted to higher temperatures with increasing EG content indicating that junction zones in PVA gels became more heat resistant with increasing EG content. This situation is different from that observed in PVA-DMSO-water gels, ${ }^{6(a)}$ where $T_{\mathrm{m}}$ as a function of DMSO content became maximum around $0.3 \mathrm{mf}$ DMSO.

Eldridge-Ferry plot $^{21}$ i.e., the logarithm of PVA concentration vs. the inverse of $T_{\mathrm{m}}$ for PVA gels with various EG contents is shown in Figure 5. The heat absorbed on forming $1 \mathrm{~mol}$ of junction zones $\Delta H_{\mathrm{m}}$ is proportional to the slope of straight lines in Eldridge-Ferry plot. As is clearly seen in Figure 5, $\Delta H_{\mathrm{m}}$ increased with increasing EG content indicating again that junction zones in PVA gels become more heat resistant with increasing EG content.

Figure 6 shows the endothermic enthalpy $\Delta H$ estimated from the area enclosed by an endothermic peak and the baseline in Figures 4(A)- $-(\mathrm{H})$ as a function of PVA concentration for various EG contents. $\Delta H$ increasesd

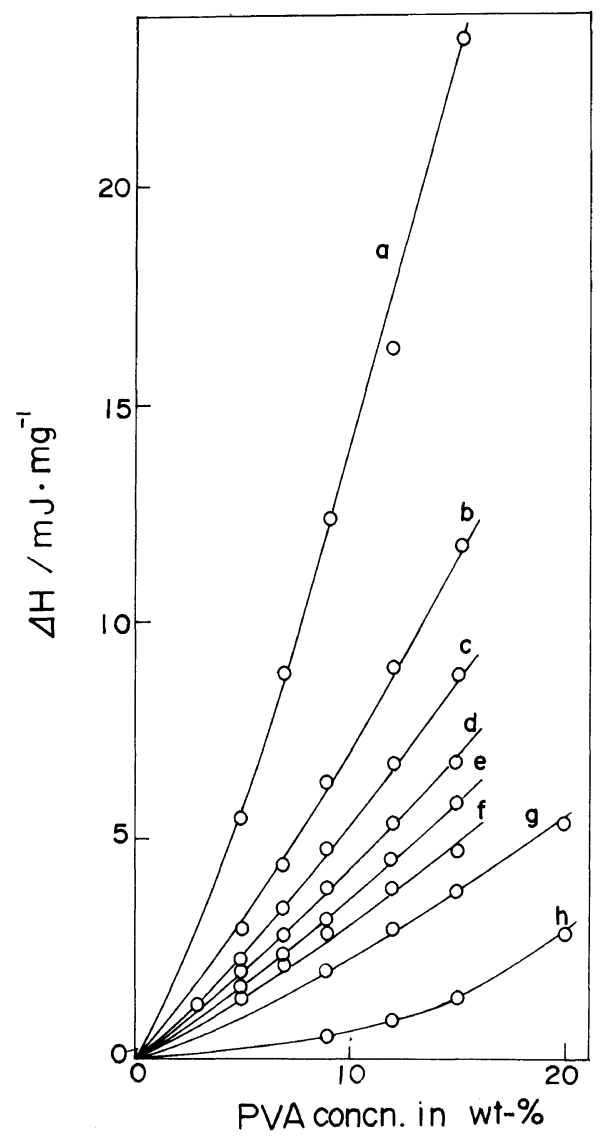

Figure 6. Endothermic enthalpy as a function of PVA concentration. EG contents: a, $1 \mathrm{mf}$; b, $0.794 \mathrm{mf}$; c, $0.458 \mathrm{mf}$; d, $0.357 \mathrm{mf}$; e, $0.222 \mathrm{mf}$;, $0.147 \mathrm{mf}$; g, $0.087 \mathrm{mf}$; h, $0.04 \mathrm{mf}$.

with increasing PVA concentration and with increasing EG content. This is again different from the case of PVA-DMSO-water gel where $\Delta H$ as a function of DMSO content became maximum around $0.3 \mathrm{mf}$ DMSO (ref $6(a))$.

Heating DSC curves from $-120^{\circ} \mathrm{C}$ to $20^{\circ} \mathrm{C}$ for $\mathrm{EG}-$ water mixtures with various EG contents are shown in Figure 7. An endothermic peak observed in water alone, in EG alone, and in mixtures with $0.087 \mathrm{mf}$, and $0.222 \mathrm{mf}$ EG is attributed to the melting of frozen water or EG. In addition to an endothermic peak, an exothermic peak appeared in mixtures with EG contents from 


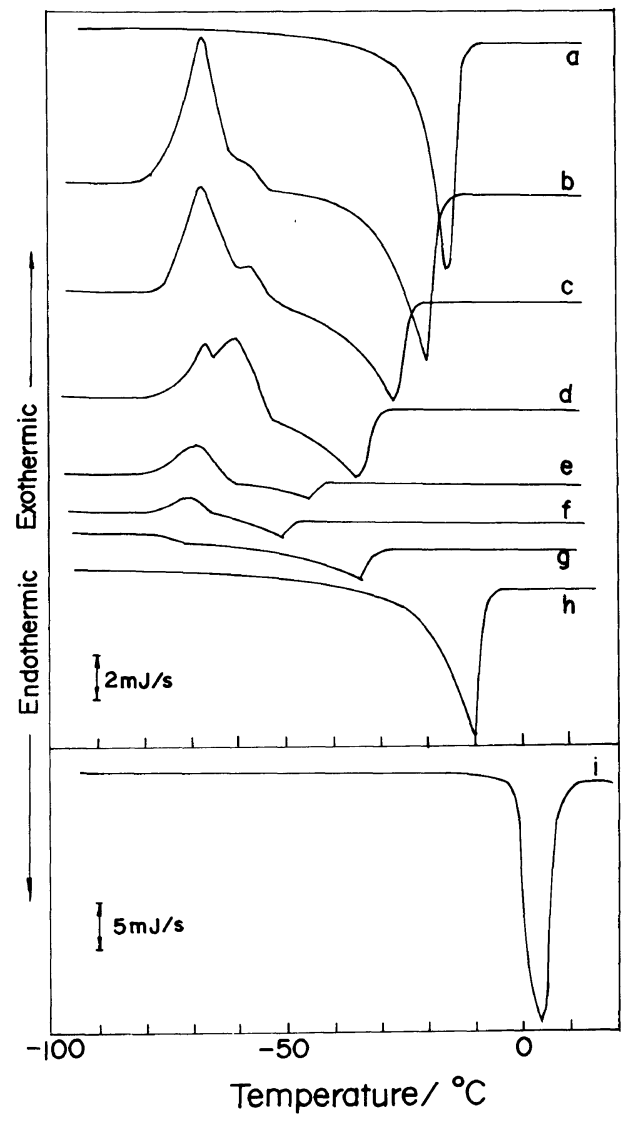

Figure 7. Heating DSC curves for EG-water systems. EG contents: a, $1 \mathrm{mf}$; b, $0.877 \mathrm{mf}$;, $0.794 \mathrm{mf}$; d, $0.668 \mathrm{mf}$; e, $0.458 \mathrm{mf}$;, $0.298 \mathrm{mf}$; g, $0.222 \mathrm{mf}$; h, $0.087 \mathrm{mf}$; i, $0 \mathrm{mf}$.

$0.298 \mathrm{mf}$ to $0.877 \mathrm{mf}$. This suggests that solvent properties of mixtures are drastically changed at around $0.3 \mathrm{mf}$ EG. The heat of fusion $\Delta H_{\mathrm{mf}}$ for frozen solvent estimated from the area enclosed by an endothermic peak and baseline in Figure 7 is shown together with the melting temperature $T_{\mathrm{mf}}$ as a function of EG content in Figure 8. The quantity of frozen solvent becomes minimum around $0.3 \mathrm{mf}$ EG. Gels consist of the balance between the solubility and the crystallinity. Below $0.3 \mathrm{mf} \mathrm{EG}$, the so-called "free water" solubilizes PVA, while above this EG content, free EG molecules solubilize PVA. Therefore, the number of junction zones seems to become maximum at $0.3 \mathrm{mf}$

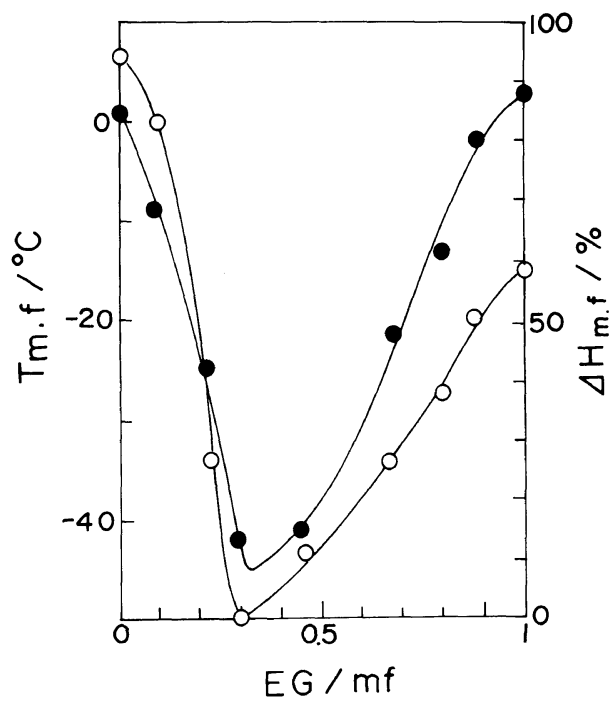

Figure 8. Melting temperature $T_{\mathrm{mf}}$ and heat of fusion $H_{\mathrm{mf}}$ as a function of EG mole fraction. $\bigcirc, T_{\mathrm{mf}} ; \boldsymbol{O}, H_{\mathrm{mf}}$.

EG content.

$E^{\prime}$ for PVA-EG-water gels $(D P=1700$, $D S=99.9 \%$, concn $=15 \%)$ is smaller than that for PVA-DMSO-water gels $(D P=1700$, $D S=99.9 \%$, concn $=15 \%$ ) reported in ref 6(a) at the optimum EG or DMSO content for gel formation at any temperature indicating DMSO is more effective in increasing the number of junction zones. However, PVA-EG without water forms a gel, whilst PVA-DMSO without water does not. Both DMSO and EG dissolve PVA, but the latter has hydroxyl groups which help to form junction zones by hydrogen bonds. DMSO shows a stronger interaction with water as has been shown by a flat heating DSC curve for a PVA-DMSO-water gel with $0.277 \mathrm{mf}$ DMSO (Figure 6 in ref 6(a)), whilst DSC curves for EG-water systems showed an endothermic peak (Figure 7) at any EG content, which was attributed to the melting of frozen solvent. Therefore, in the presence of water, DMSO can make stronger gels than EG because it decreases much more free water than EG. 


\section{REFERENCES}

1. H. Maeda, T. Kawai, and R. Kashiwagi, Kobunshi Kagaku (Chem. High Polymers, Japan), 13, 193 (1956).

2. M. Watase and K. Nishinari, Polymer, 24, 270 (1983).

3. M. Watase and K. Nishinari, Makromol. Chem., 189, 871 (1988).

4. L. V. Domotenko, V. I. Lozinskii, Ye. S. Vainerman, and S. V. Rogozhin, Vysokomolekul. Soed. Ser. A, 30, 1661 (1988).

5. F. Yokoyama, I. Masada, K. Shimamura, T. Ikawa, and K. Monobe, Colloid Polym. Sci., 264, 595 (1986).

6. M. Watase and K. Nishinari, Polym. J., 21, (a) 567 and (b) 597 (1989).

7. S. Okada, H. Hoshino, H. Urakawa, K. Kajiwara, and T. Ito, Polym. Prepr. Jpn., 37 (No.9), 3064 (1988).

8. K. Nishinari and M. Watase, Agric. Biol. Chem., 51, 3231 (1987).

9. M. Watase, K. Kohyama, and K, Nishinari, Thermochim. Acta, 206, 163 (1992).
10. K. Nishinari, S. Koide, P. A. Williams, and G. O. Phillips. J. Phys. France, 51, 1759 (1990).

11. T. Matsuo and H. Inagaki, Makromol. Chem., 53, 130 (1962).

12. D. Braun and E. Walter, Colloid Polym. Sci., 258, 376 (1980).

13. T. Takigawa, H. Kashihara, K, Urayama, and $\mathrm{T}$. Masuda, Polymer, 33, 2334 (1992).

14. K. Nishinari, S. Koide, and K. Ogino, J. Phys. France, 46, 793 (1985).

15. L. R. G. Treloar, "The Physics of Rubber Elasticity," Clarendon Press, Oxford, 1975.

16. K. Nishinari, M. Watase, and K. Ogino, Makromol. Chem., 185, 2663 (1984).

17. A. H. Clark and S. B. Ross-Murphy, Br. Polym. J., 17, 164 (1985).

18. D. Oakenfull, J. Food Sci., 49, 1103 (1984).

19. A. H. Clark and S. B. Ross-Murphy, Adv. Polym. Sci., 83, 57 (1987).

20. M. Watase and K. Nishinari, Carbohydr. Polym., 11, 55 (1989).

21. J. E. Eldridge and J. D. Ferry, J. Phys. Chem., 58, 992 (1954). 